\title{
Short-term effect of kinesiology taping on pain, functional disability and lumbar proprioception in individuals with nonspecific chronic low back pain: a double-blinded, randomized trial
}

\author{
Soheila Abbasi ${ }^{1}$, Mohammad-Reza Hadian Rasanani ${ }^{2 *}$, Nastaran Ghotbi ${ }^{1}$, Gholam Reza Olyaei ${ }^{1}$,
} Ali Bozorgmehr ${ }^{3}$ and Omid Rasouli ${ }^{4}$

\begin{abstract}
Background: This study aimed to evaluate the effect of kinesiology taping (KT) on lumbar proprioception, pain, and functional disability in individuals with nonspecific chronic low back pain (CLBP).

Methods: Thirty individuals with nonspecific CLBP participated in this double-blinded, randomized clinical trial from July 2017 to September 2018. The participants were randomized into two groups: KT $(n=15)$ and placebo group $(n=15)$. KT was applied with $15-25 \%$ tension for $72 \mathrm{~h}$, and placebo taping was used without tension. Lumbar repositioning error was measured by a bubble inclinometer at three different angles $\left(45^{\circ}\right.$ and $60^{\circ}$ flexion, and $15^{\circ}$ extension) in upright standing. Pain and disability were assessed by the Short-Form McGill Pain Questionnaire and Oswestry Disability Index, respectively. All measurements were recorded at baseline and 3 days after taping.
\end{abstract}

Results: Pain and disability scores reduced 3 days after taping in the KT group with large effect sizes $(p<0.05)$. Only the total score of pain was significantly different between the groups 3 days after taping and improved more in the KT group with a large effect size $(p<0.05)$. However, lumbar repositioning errors were similar between the groups after 3 days $(p>0.05)$. Also, only constant error of $15^{\circ}$ extension showed a moderate correlation with disability $(r=$ $0.39, p=0.02)$.

Conclusion: KT can decrease pain and disability scores after 3 days of application. Although placebo taping can reduce pain, the effect of $K T$ is higher than placebo taping. The findings do not support the therapeutic effect of $K T$ and placebo taping as a tool to enhance lumbar proprioception in patients with nonspecific CLBP.

Trial registration: The study prospectively registered on 21.05.2018 at the Iranian Registry of Clinical Trials: IRCT2 0090301001722N20.

Keywords: Pain level, disability, Proprioception, Low Back pain, Kinesiotape, Sham

\footnotetext{
*Correspondence: hadianrs@sina.tums.ac.ir; hadianrasan@gmail.com

${ }^{2}$ Postgraduate Department, School of Rehabilitation, Tehran University of

Medical Sciences, International, Brain and Spinal Injury Research Center

(BASIR), Institute of Neuroscirnce, P.O. Box: 111551683, Tehran, Iran

Full list of author information is available at the end of the article
}

C C The Author(s). 2020 Open Access This article is licensed under a Creative Commons Attribution 4.0 International License, which permits use, sharing, adaptation, distribution and reproduction in any medium or format, as long as you give appropriate credit to the original author(s) and the source, provide a link to the Creative Commons licence, and indicate if changes were made. The images or other third party material in this article are included in the article's Creative Commons licence, unless indicated otherwise in a credit line to the material. If material is not included in the article's Creative Commons licence and your intended use is not permitted by statutory regulation or exceeds the permitted use, you will need to obtain permission directly from the copyright holder. To view a copy of this licence, visit http://creativecommons.org/licenses/by/4.0/. The Creative Commons Public Domain Dedication waiver (http://creativecommons.org/publicdomain/zero/1.0/) applies to the data made available in this article, unless otherwise stated in a credit line to the data. 


\section{Background}

It is known that proprioception is necessary for the control of human movement [1]. Proprioception is described as the joint's sensation, position, and movement; also, the sense of force, effort, weight, and perceived timing associated with muscular contraction [2]. Proprioceptive inputs are derived from afferent information received from muscle, joint, and skin receptors [2]. These receptors have different roles depending on the range at a given joint; for example, previous studies have found that joint receptors to be activated near the end of the joint range, while muscle spindles provide afferent inputs throughout the physiologic ranges [3].

It is challenging to measure proprioception because of its complex function. In previous studies, proprioception has been measured by force-plate analysis [4], electromyographic (EMG) activity [5], and position sense [6]. Position sense is defined as the ability to perceive the movement or orientation of a body segment in space. Some studies have used repositioning error (RE) or position sense to measure proprioception in joints $[6,7]$. There is evidence that individuals with chronic low back pain (CLBP) have reduced proprioceptive ability and larger lumbar $\mathrm{RE}$ in the lumbar region $[7,8]$, but some studies have found no significant difference between subjects with CLBP with pain-free participants [9, 10]. If proprioceptive deficits exist, rehabilitation programs should be designed to improve proprioception; however, there has been little research to support this.

In recent years, the use of a therapeutic tool called kinesiology tape (KT) has increasingly become popular to use for musculoskeletal disorders. KT is made up of colorful elastic cotton strips with an acrylic adhesive that may be stretched to up to $140 \%$ of their original length [11]. KT is assumed to have several benefits [12], including (1) pain reduction through neurological suppression, (2) reposition of subluxated joints by decreasing abnormal muscle tone, (3) to create more space by lifting fascia and soft tissue to improve circulation, (4) correcting muscle function by strengthening weak muscles (5) and providing sensory stimulation to improve proprioception $[12,13]$.

The compressive and stretching effect of $\mathrm{KT}$ provides additional cutaneous stimulations, and these stimulations transfer more information regarding the joint position and movement to the central nervous system (CNS) for integration resulting in increased proprioception $[2,14]$. However, there have been controversial results regarding the effect of $\mathrm{KT}$ on proprioception.

Some studies have shown improved proprioception through augmented cutaneous sensory stimulations provided by $\mathrm{KT}[14,15]$, whereas others have found no changes in proprioception using KT $[13,16]$. Therefore, CLBP individuals with poor proprioception might benefit from the application of $\mathrm{KT}$.

As yet, there is a lack of research exploring the effect of KT on lumbar proprioception. The purpose of the present study was to explore the short-term effect of KT on lumbar proprioception, pain, and functional disability in individuals with nonspecific CLBP. The associations between pain intensity, disability, and lumbar RE were also evaluated in these individuals. It was hypothesized that applying $\mathrm{KT}$ on the lumbar spine would improve lumbar proprioception, pain, and disability compared to the placebo group in individuals with nonspecific CLBP.

\section{Methods \\ Study design}

This study was a parallel double-blinded (assessor and participants), prospective randomized trial. The study was conducted at the Tehran University of Medical Sciences in accordance with the principles of the Declaration of Helsinki. The Ethical Committee at the Tehran University of Medical Sciences has approved this study, and it was prospectively registered at the Iranian Registry of Clinical Trials: IRCT20090301001722N20 at the website https://www.irct.ir/. All participants signed a consent form before entering the study.

\section{Participants}

A total of 43 individuals were screened for this study. Thirteen were excluded for the reasons presented in Fig. 1. Thus, 30 individuals with nonspecific CLBP (15 males, 15 females), ages 25-50 years, participated in the study. All participants were referred from two public physical therapy clinics associated with the Tehran University of Medical Sciences from July 2017 to September 2018. Based on the definition of nonspecific CLBP, the inclusion criteria were localized back pain between the 12th rib and the gluteal folds lasting more than 3 months. The individuals were excluded if they had any of the following criteria: the history of pain radiating further than the buttock, sciatica or other radicular involvement, spinal surgery, nerve root compression, neurological deficits, rheumatic diseases, diabetes, mental disorders, pregnancy, lower extremity injuries, or neuromuscular diseases. Participants were also excluded if they presented signs of allergy to KT during a test conducted before the initial evaluation. KT without tension was used to test if volunteers had any allergic reactions to taping, and three individuals (of 43 individuals) had allergic reactions, e.g. redness and itching, to KT; therefore, they were excluded from the study. No one showed any allergic reactions before or after the removal 


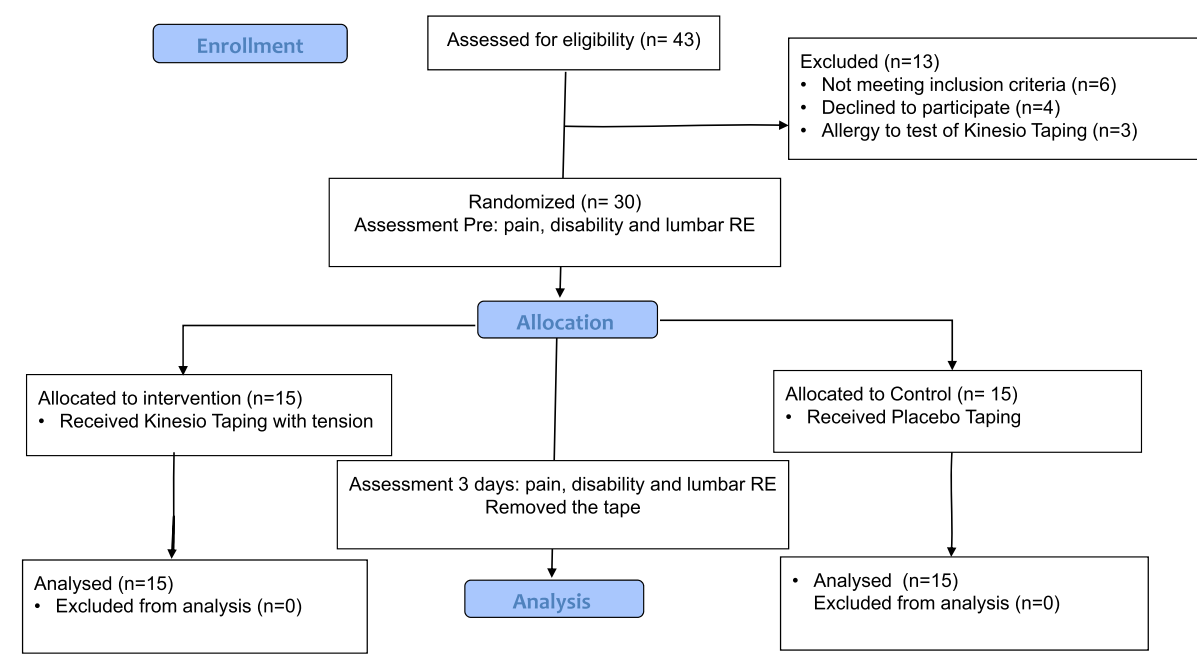

Fig. 1 The flow of participants recruitment through the study

of the tape among those 30 participants who completed the testing.

\section{Procedure}

Individuals were randomly assigned to either placebo group (KT without tension) or experimental group (KT with tension) according to a randomization scheme generated by a computer. Individuals with odd numbers were allocated to the placebo group, and individuals with even numbers were allocated to the KT group. The allocation of the participants was concealed by using sequentially numbered, sealed, and opaque envelopes. Blinded investigator (investigator 1) conducted the data collection and analysis, and investigator 2 conducted enrolling participants and intervention to minimize potential sources of bias. Also, all participants were unaware of their group. Figure 1 displays the recruitment process for this study. All outcomes were collected in a biomechanics laboratory at the Tehran University of Medical Science.

\section{Intervention and placebo group}

In this study, waterproof, adhesive tape (NST-05002, made in Korea) with a width of $5 \mathrm{~cm}$ and a thickness of $0.5 \mathrm{~mm}$ was used. The experimental group received a standardized KT application in sitting position for $72 \mathrm{~h}$. We had chosen applying this method of KT (star shape) in sitting position following the method used by previous similar studies $[17,18]$. Four I-strips, including one vertical, one horizontal, and two at $45^{\circ}$ angles to vertical strip, were attached with 15-25\% tension overlapping in a star shape over the point of maximum pain in the lumbar area. The central part of the strips was applied before the ends by pressing and adhering, and all strips have crossed at the central point of the tape (Fig. 2a). It seems that the star shape of KT with 15-25\% tension in the lumbar region is more effective than other methods in reducing pain and disability and stimulation of mechanoreceptor in subjects LBP $[12,18,19]$.

The placebo group received a single I-strips of the same tape without tension transversely on the maximum pain point of the lumbar region (placebo taping) (Fig. 2b). After 3 days, the taping was removed for both groups, and re-evaluation was performed by investigator 1 .

\section{Outcomes measures}

Assessments were taken at baseline and 3 days after the intervention. All participants completed measures of pain intensity by the Short-Form McGill Pain Questionnaire (SF-MPQ) [20], functional disability by Oswestry Disability Index (ODI) [21], and lumbar RE using bubble inclinometer before and after the intervention.

The SF-MPQ consists of three parts, including 15 descriptors of pain (11 from sensory categories, and 4 from affective categories), Visual Analogue Scale (VAS), and present pain intensity (PPI). Descriptors are rated based on pain severity on a four-point scale $(0=$ none, $1=$ mild, $2=$ moderate, $3=$ severe). The scores of sensory and affective (S/A) part are calculated by summing sensory and affective item values, as the total score. The second part is VAS, which is a $10-\mathrm{cm}$ horizontal line ranging from "no pain" to "worst possible pain." Patients present the severity of their pain by marking the line. After that, pain intensity was calculated from zero to the marked point by the patient in centimeters. The third part of the SF-MPQ is the PPI, which is a six-point verbal rating scale ranging from none $(0)$ to the worst excruciating (5). The patients choose the word that best describes the overall intensity of their pain at the present time; then, 

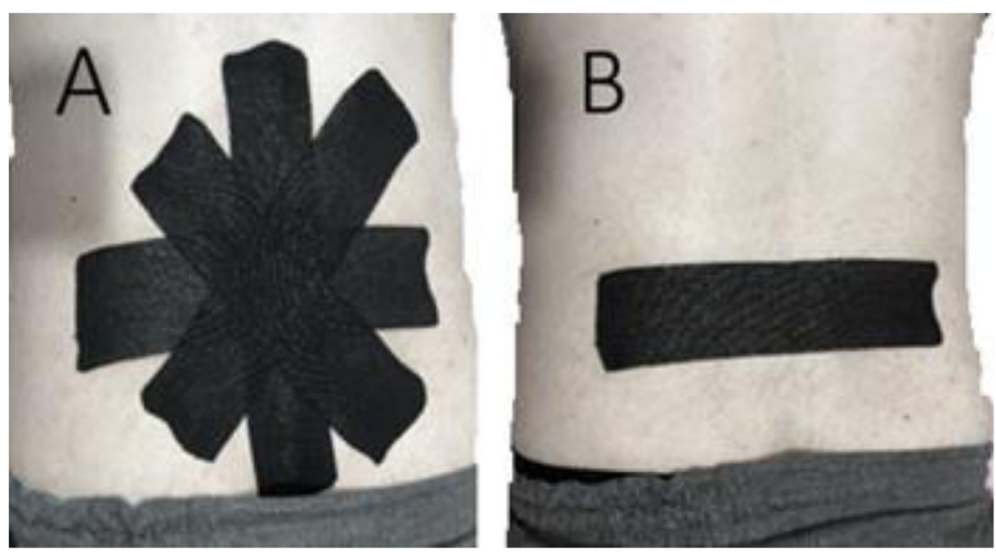

Fig. 2 Star shape Kinesio tape application that four I-strips of KT was placed over the point of maximum pain in the lumbar area (a), placebo taping that single I-strip applied transversely immediately above the point of maximum lumbar pain (b)

the questionnaire is completed. The MPQ as a multidimensional tool is designed to assess sensory, affective, and evaluative dimensions of pain [20].

Considering the effects of KT on various aspect of pain (i.e., sensory, affective and evaluative dimensions of pain) [22], it seems that the MPQ could better show the effects of KT on pain after taping than other tools such as VAS alone or Numerical Rating Scale (NRS). Also, the conflicting results about the effects of KT on pain in individuals with LBP probably resulted from it. The current study was the first study that investigated the effects of KT on pain using SF-MPQ as a multidimensional tool in subjects with nonspecific CLBP.

In addition, lumbar RE, as an indirect measure of proprioception, was determined using a bubble inclinometer because of its reasonable price, the facility of application, accessibility, and non-invasiveness. Results of previous studies showed that measuring the lumbar range of motion (ROM with a bubble inclinometer was valid and reliable [23-25]. The bubble inclinometer is circular, fluidfilled disc devices, with an adjustable scale to permit zeroing (Model 10,602 built by Fabrication Enterprise Inc. USA) (Fig. 3).

The participant wore only shorts to reduce sensory cues from clothing. First, the therapist marked the T12L1 and S1 spinal levels by a marker. Two bubble inclinometers were used simultaneously for measuring the flexion and extension of lumbar ROM in standing position. The participant stood with feet $15 \mathrm{~cm}$ apart and arms at their sides while looking forward. One inclinometer was placed at T12-L1, and the other one located over the sacrum. The inclinometers were set as close to 0 degrees as possible. While holding the inclinometers, the participant was asked to bend forward and keep the knees straight. Maximum values in both inclinometers were recorded. The actual range of Lumbar flexion was calculated by subtracting the records from $\mathrm{S} 1$ from the device placed over T12-L1 [23].

The same landmarks and procedures that were defined for the flexion technique were used for measuring lumbar extension. Both inclinometers were located over the skin marks while holding the inclinometers, and the therapist instructed the participant to bend backward. The angles were recorded on the inclinometers and subtracted [23].

Repositioning accuracy was assessed with participants trying to reproduce the target position.

Three different target position was chosen. Participants were asked to reproduce two different trunk positions from the neutral spinal posture to $45^{\circ}$ and $60^{\circ}$ flexion and one position from a neutral spinal posture to $15^{\circ}$ extension. These angles were chosen because previous research indicated that participants with LBP have greater difficulties in reproducing $45^{\circ}$ and $60^{\circ}$ lumbar flexion and $15^{\circ}$ lumbar extension than healthy ones $[8,26]$.

Each participant was positioned into an upright neutral starting position. This position was such that the anterior superior iliac spine and the posterior superior iliac spine were aligned in the horizontal plane using a pelvic inclinometer. To determine the maximum available lumbar ROM and whether the participant was able to perform the experimental tasks, each participant was instructed to move into flexion as much as possible, then the participant moved into extension as much as possible. Each participant was positioned in $45^{\circ}$ of lumbar flexion for $10 \mathrm{~s}$ and was asked to remember the position because he or she would be asked to reproduce this position with closed eyes. Next, the participant returned to the neutral position and then was given the verbal instruction of reproducing the target position as accurately as he or she could. The participant reported to the 


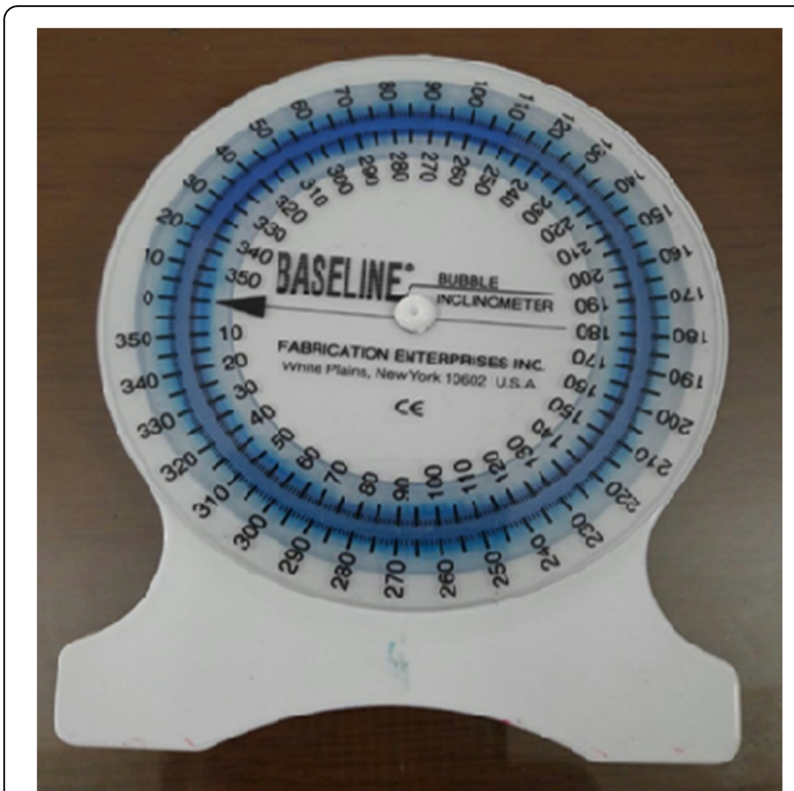

Fig. 3 The bubble inclinometer

therapist on reaching the target position as perceived by him or her. The participant was required to hold the final position for $3 \mathrm{~s}$; then, the reproduced position was recorded by the therapist. The same procedures were defined for $60^{\circ}$ lumbar flexion and $15^{\circ}$ lumbar extension. The tests were randomly performed, and each test was repeated three times with a $30 \mathrm{~s}$ rest between each trial; then, the average of them was calculated for the analysis. The participants were allowed to practice the test protocol once before the test. No feedback was given during testing.

At baseline, all outcome measures were assessed for each participant before the intervention. Then, the participants were taped, and re-evaluation was performed after 3 days. RE was defined as the constant error (CE) between the target position and the actual measured angles that $\mathrm{CE}$ indicates error towards a particular direction (positive or negative and the absolute error (AE) was the unsigned deviation from the target position, thus reflecting absolute repositioning error, irrespective of error direction. Both errors were assessed before and after the intervention.

\section{Statistical analysis}

The sample size calculation was performed using G*Power version 3.1.9.2 [27]. This was determined using a power of 0.8 , an alpha value of 0.5 . It resulted in a required sample size of 13 subjects in each group. Assuming an attrition rate of $10 \%$ due to dropouts and technical problems, it was determined that we would require 15 participants in each group.
Statistical analyses were performed using IBM SPSS Statistics for Windows, version 22.0 (IBM Corp., Armonk, NY, USA). The normality of data was assessed, and all variables followed a normal distribution. In this study, all variables (pain, functional disability, CE, and $\mathrm{AE}$ values) were presented as mean (standard deviation). All variables were compared between the groups using independent sample t-tests. Paired t-tests were also applied to find the differences between before and 3 days after the taping in each group. Cohen $d$ was reported as the estimates of effect size, with the classification of small $(d=0.2)$, medium $(d=0.5)$, and large $(d \geq 0.8)$ [28]. The correlations between pain intensity, disability, and RE were assessed using Pearson correlation coefficient before taping for all participants. The correlations were rated as strong $(>0.7)$, moderate $(>0.5)$, or weak $(<0.3)$ [29]. The level of significance was set at $p<0.05$.

\section{Results}

The demographic characteristics of the participants are shown in Table 1. The participants in the placebo and KT groups had a mean age of 42 and 44 years, respectively, with mild to moderate pain and disability. Half of the participants were females. After randomization, all participants were assessed, and none of the participants were excluded from the study. The groups were comparable at baseline, and there were no significant differences between the two groups regarding age, gender, BMI, pain (Total score of pain (S/A), VAS, PPI), or disability (ODI) (Table 1).

\section{Effect of intervention}

There were no significant differences in pain (total score of pain (S/A), VAS and PPI), functional disability or RE $(\mathrm{AE}$ and $\mathrm{CE})$ at the angles $\left(45^{\circ}\right.$ and $60^{\circ}$ flexion and $15^{\circ}$ extension) between the groups at baseline $(P>0.05)$. After 3 days of taping, the pain (total score of pain (S/A), VAS, and PPI) and functional disability scores significantly reduced in the KT group with large effect sizes $(\mathrm{d}>0.8)$, also PPI significantly decreased in the placebo group with a large effect size $(P<0.05, \mathrm{~d}=0.8)$. However, the difference between the groups was significant only for the total score of pain (S/A) 3 days after taping and improved significantly more in the KT group than the placebo group with large effect size $(P<0.05)$ (Table 2).

No significant between-group or within-group differences were observed for the RE ( $\mathrm{AE}$ and $\mathrm{CE}$ ) in any of the angles $\left(45^{\circ}\right.$ and $60^{\circ}$ flexion and $15^{\circ}$ extension) at 3 days $(P>0.05)$ (Table 2$)$.

\section{Correlations with RE}

According to the findings, only $\mathrm{CE}$ of $15^{\circ}$ extension showed a significant moderate positive correlation with disability $(\mathrm{r}=0.39, P=0.02)$ (Table 3 ). 
Table 1 Characteristics of participants in each group

\begin{tabular}{llll}
\hline Variable & $\begin{array}{l}\text { Kinesio taping } \\
(\boldsymbol{n}=\mathbf{1 5})\end{array}$ & $\begin{array}{l}\text { Placebo } \\
(\mathbf{n}=\mathbf{1 5})\end{array}$ & $\boldsymbol{P}$-value \\
\hline Age (years) & $44.3 \pm 3.6$ & $42.1 \pm 6.9$ & $0.274^{\dagger}$ \\
Gender (female) & $9(60 \%)$ & $6(40 \%)$ & $0.439^{\ddagger}$ \\
BMI (kg/m2) & $24.9 \pm 3.5$ & $24.8 \pm 3.6$ & $0.890^{\dagger}$ \\
Total score of pain (S/A) & $18.2 \pm 6.9$ & $15.4 \pm 5.4$ & $0.296^{\dagger}$ \\
Pain (VAS) & $5.6 \pm 1.4$ & $4.7 \pm 1.1$ & $0.062^{\dagger}$ \\
PPI & $2.2 \pm 0.7$ & $2.1 \pm 0.4$ & $0.549^{\ddagger}$ \\
Disability (ODI) & $21.1 \pm 6.1$ & $19.2 \pm 6.1$ & $0.397^{\dagger}$ \\
\hline
\end{tabular}

BMI Body Mass Index, VAS Visual Analogue Scale, ODI Oswestry Disability Index, on a percentage scale, score from 0 to $20 \%$ indicate a minimal disability, $21-40 \%$ indicate a moderate disability, $41-60 \%$ severe disability, $61-$ $80 \%$ for crippled and $81-100 \%$. S/A Sensory and Affective, PPI Present Pain Intensity, $A E$ Absolute Error, $C E$ Constant Error. Data are presented as means and standard deviation (Mean \pm SD). † Independent sample t-test, $\neq$ Chi-square test

\section{Discussion}

The current study aimed to examine the effect of KT application on lumbar proprioception, pain, and functional disability in individuals with nonspecific CLBP. The results showed that pain (Total score (S/A), VAS and PPI) and disability scores significantly improved after 3 days of taping in the KT group with large effect sizes, but no significant differences in $\mathrm{RE}$ ( $\mathrm{AE}$ or $\mathrm{CE}$ ) were found in any of the three angles $\left(45^{\circ}\right.$ and $60^{\circ}$ flexion and $15^{\circ}$ extension). In the placebo group, only PPI was significantly reduced after 3 days of placebo taping without tension. Also, only the total score of pain (S/A) showed significant differences between the groups with a large effect size.

Participants in the KT group had mild to moderate pain levels and showed approximately $23 \%$ reduction of pain over 3 days; therefore, possibly over a longer period, a greater reduction would have been achieved. However, $\mathrm{KT}$ is typically worn for $3-5$ days.

There are several studies investigated the effect of KT on pain and disability in individuals with LBP [17, 30, 31]; however, there is a large variability in a combination of KT with other therapies, such as exercise program, manual therapy, and traditional physical therapy. Some studies compared the effect of KT to placebo taping on pain and disability. In line with our findings, some studies showed that the levels of pain and disability significantly decreased after taping [18, 32-34], and some reported that $\mathrm{KT}$ is better than placebo taping in patients with CLBP $[32,34,35]$. On the other hand, some studies found no significant decrease in pain or disability after the intervention [36, 37], and that the application of KT was not better than placebo taping for patients with CLBP [31, 33, 37], which is contradicted with our findings. In the current study, similar to previous studies $[18,32,33,35,36]$, the placebo taping had therapeutic effectiveness beyond the placebo. It seems that applying placebo taping without tension even with one strip can create some physiological.In the current study, KT only reduced the total score of pain (S/A) in the KT group compared to the placebo group. It indicates that KT can affect other aspects of pain (i.e., sensory, affective) that placebo taping could not affect. Because of the PPI reduction in the placebo group, it seems that the application of placebo taping could reduce pain intensity due to a placebo effect in the patients with CLBP. Based on our results, both $\mathrm{KT}$ and placebo taping can reduce pain in patients with CLBP, but the effects of KT are higher than placebo taping.

Some physiological mechanisms of KT effects have been proposed. Pain reduction after applying KT with tension may be due to lifting the skin and enhancing subcutaneous space and, as a result, reduced activation of pain receptors, also possibly activates descending inhibitory system. Also, in the gate control theory of pain, tactile stimulation of KT would reduce the afferent signal of large-diameter non-nociceptive fibers resulting in a reduction of pain [18, 38, 39]. In addition, some positive effects of KT may indirectly affect pain and disability improvement, such as normalize muscle tone, improvements of postural control, range of motion, circulation, and proprioception [40-43].

The previous studies never evaluated the effects of KT on pain using SF-MPQ as a multidimensional tool in individuals with nonspecific CLBP, and they measured pain by VAS $[18,32,34]$ or NRS [33, 35-37]. The current study is the first to assess the effects of KT on pain using SF-MPQ in individuals with nonspecific CLBP.

Also, there were no significant differences (betweengroup or within-group) for lumbar $\mathrm{RE}$ ( $\mathrm{AE}$ and $\mathrm{CE}$ ) in the angles $\left(45^{\circ}\right.$ and $60^{\circ}$ flexion and $15^{\circ}$ extension). To the best of our knowledge, this is the first study to evaluate the effect of KT on lumbar proprioception in patients with LBP. Several studies have evaluated the effect of KT on proprioception in peripheral joints, especially knee and ankle in injured or noninjured individuals $[11,13-15]$ that are conflicting with our results. Several explanations may explain our results. Based on our results, it seems that placebo taping may affect and reduce pain, albeit slightly. Therefore, the lack of non-taping group and the effect of placebo taping may have affected the results of between-group comparison in this study.

A systematic review reported that patients with LBP have impaired lumbar proprioception compared with controls when measured actively in sitting positions [4]. In our study, lumbar proprioception was actively measured in standing positions that may have affected the results. Also, various methods of measuring lumbar proprioception exist, including joint RE, the threshold to 


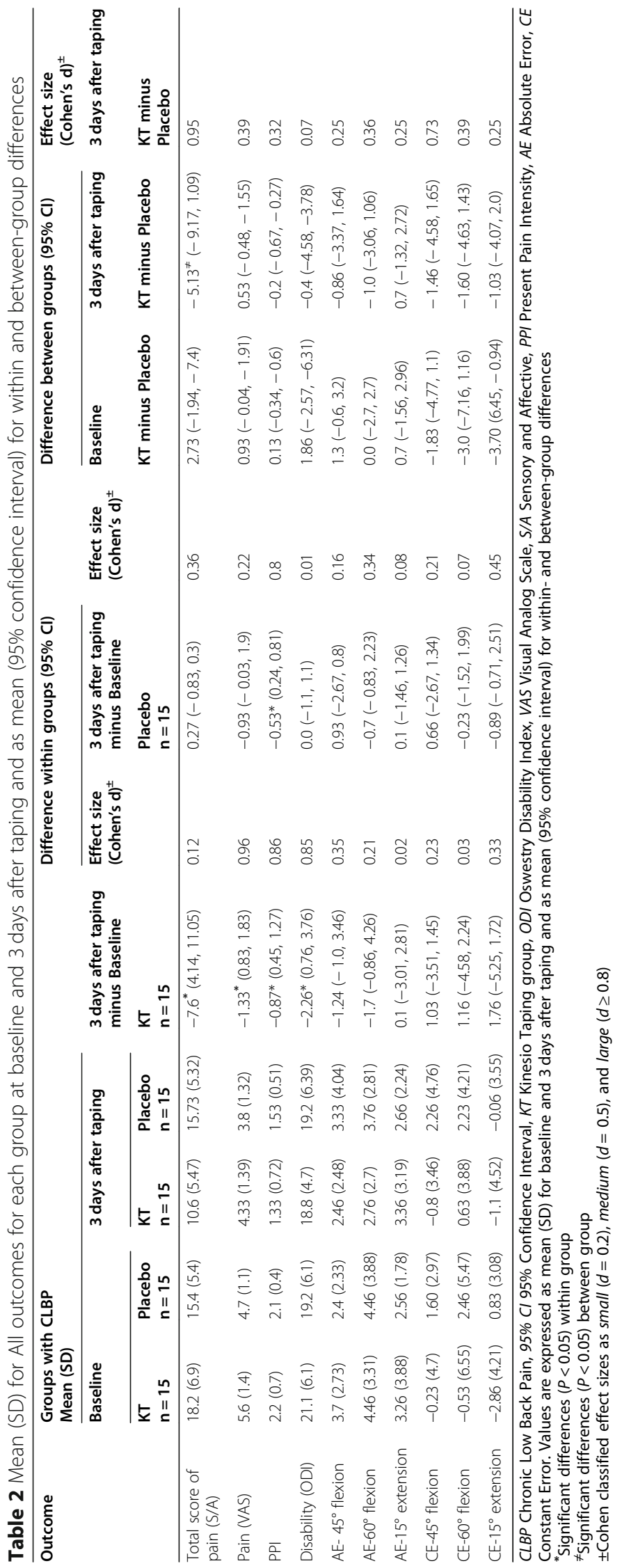


Table 3 The correlations between repositioning error values (constant error and absolute error), pain, and disability

\begin{tabular}{|c|c|c|c|c|}
\hline & Pain (Total score of pain) (S/A) & Pain (VAS) & Pain (PPI) & Disability (ODI) \\
\hline \multicolumn{5}{|l|}{ CE } \\
\hline \multirow[t]{2}{*}{$45^{\circ}$ flexion } & $r=0.14$ & $r=-0.03$ & $r=-0.12$ & $r=-0.15$ \\
\hline & $p=0.435$ & $p=0.853$ & $p=0.501$ & $p=0.427$ \\
\hline \multirow[t]{2}{*}{$60^{\circ}$ flexion } & $r=-0.04$ & $r=-0.17$ & $r=-0.12$ & $r=-0.11$ \\
\hline & $p=0.815$ & $p=0.359$ & $p=0.513$ & $p=0.560$ \\
\hline \multirow[t]{2}{*}{$15^{\circ}$ extension } & $r=0.19$ & $r=-0.32$ & $r=-0.06$ & $r=0.39$ \\
\hline & $p=0.293$ & $p=0.080$ & $p=0.721$ & $p=0.029^{*}$ \\
\hline \multicolumn{5}{|l|}{$A E$} \\
\hline \multirow[t]{2}{*}{$45^{\circ}$ flexion } & $r=-0.23$ & $r=0.12$ & $r=-0.30$ & $r=-0.16$ \\
\hline & $p=0.216$ & $p=0.50$ & $p=0.106$ & $p=0.377$ \\
\hline \multirow[t]{2}{*}{$60^{\circ}$ flexion } & $r=-0.33$ & $r=0.22$ & $r=0.20$ & $r=0.17$ \\
\hline & $p=0.074$ & $p=0.234$ & $p=0.282$ & $p=0.366$ \\
\hline \multirow[t]{2}{*}{$15^{\circ}$ extension } & $r=-0.10$ & $r=-0.02$ & $r=-0.17$ & $r=-0.23$ \\
\hline & $p=0.601$ & $p=0.912$ & $p=0.366$ & $p=0.209$ \\
\hline
\end{tabular}

ODI Oswestry Disability Index, VAS Visual Analog Scale, S/A Sensory and Affective, PPI Present Pain Intensity, AE Absolute Error, CE Constant Error

* Statistically significant: $(P$-value $<0.05)$

detection of passive motion, and directional motion perception [4]. In the current study, lumbar proprioception was measured by repositioning error. However, the best method of measuring proprioception is still unclear. In addition, in the previous studies, target positions for repositioning ranged from neutral lumbar spinal posture to target angles in pelvic tilting and lumbar flexion, extension, lateral flexion, and rotation in patients with LBP $[4,6,7]$. This study reproduced two different trunk positions from neutral to $45^{\circ}$ and $60^{\circ}$ flexion and one position from neutral to $15^{\circ}$ extension. Perhaps within other ranges, individuals show more proprioception deficits and/or improvements after KT.

The potential mechanism by which KT improves proprioception is not yet understood. Some authors have hypothesized that cutaneous feedback supplied by KT could be increased. Applied pressure and stretching due to KT application on the skin at extremes of motion, similar to joint mechanoreceptors, can also stimulate cutaneous mechanoreceptors and signal information of joint movement or joint position [13, 14, 44]. Konishi et al. (2013) confirmed that KT could counter quadriceps femoris weakness due to attenuated la afferent activity [44].

It seems that tactile stimulation of KT was not enough for improvements of proprioception may be due to short-term assessment or method of taping in our study. There are direct relationships between impaired proprioception, pain, and reduced quality of life [45]. In the current study, pain and disability improved in the patients; therefore, there would be improvements in proprioception as well as pain and disability reduction in the KT group.
It has been proposed that proprioceptive deficits may lead to trunk muscle dysfunction also may cause alterations in normal afferent inputs from the affected muscles. In neutral posture, muscle afferents could be considered as primary contributors to position sense because ligaments are under minimal tension $[3,5]$. In contrast, previous studies reported that KT could normalize lumbar muscle function and postural control in patients with LBP $[17,41,43]$, and it is thus expected to improve lumbar proprioception.

The tension of KT is described as one of the critical factors for successful implementation. Theoretically, both 75 and $100 \%$ of tension are used to support weak muscle or correction of joint position, $25-50 \%$ for muscle activation in weak muscles positioned from the origin to the muscle insertion, $15-25 \%$ for muscle inhibition caused by overuse or muscle overstretching placed from the insertion towards the muscle origin, and 0 $15 \%$ for reduction of edema [12]. The best tension of KT to improve proprioception is not evident yet. In the current study, we used the star shape of KT with 15$25 \%$ tension because it was reported that the star shape of KT with $15-25 \%$ tension significantly improved pain, disability, trunk muscle endurance, and trunk flexion range of motion in patients with nonspecific CLBP [4]. A different method of KT with different tension may enhance lumbar proprioception in patients with CLBP.

Besides, the moderate positive correlation between $\mathrm{CE}$ of $15^{\circ}$ extension and disability in these patients indicates that those with higher disability display a greater RE. Previous research suggests that spinal RE is the largest among individuals with higher disabilities. Also, there is a positive correlation between 
functional disability and $\operatorname{RE}[6,9]$. Those with a higher functional disability may have greater overall disruption of the pain neuromatrix within the CNS [6]. The reasons for nonsignificant correlations between functional disability, pain, and both $\mathrm{CE}$ and $\mathrm{AE}$ at other angles are unclear. Motor control impairments in spinal posture and movement, as well as trunk muscle activation, have been reported among patients with nonspecific CLBP $[6,43]$. Thus, these changes may explain significant or nonsignificant correlations between functional disability, pain, and both $\mathrm{CE}$ and $\mathrm{AE}$ in the three measured angles, since $\mathrm{RE}$ is influenced by muscle spindle feedback [7] and spinal posture [6].

\section{Limitations}

In the current study, some limitations should be considered while interpreting the findings. First, we compared the effects of KT with a placebo group, but the placebo taping was not a real placebo due to the volume of tape used, which should be thinner than KT with less stimulation of subcutaneous afferent sensory fibers. Also, the lack of a non-taping group is another limitation of the current study. Moreover, we only examined the shortterm effects of star shape taping with 15-25\% tension; however, higher tensions may have different effects on lumbar RE. Therefore, future studies should compare different methods of taping with non-taping, also assess longer follow-ups in patients with CLBP.

\section{Clinical implication}

The use of KT would be beneficial to decrease the level of pain and disability in individuals with nonspecific CLBP. Also, taping without tension even with one strip, such as placebo taping, may reduce pain in these patients. Since the placebo treatments are important tools that can be used by the medical community to complement regular therapies, the use of placebo taping can be helpful. Considering lumbar RE may assist therapists in identifying poor posture awareness and proprioception impairments among these patients.

\section{Conclusion}

The findings suggest that star-shaped KT reduces pain and disability scores after 3 days of application with large effect size in patients with nonspecific CLBP. It also seems that KT may affect another aspect of pain (i.e., sensory, affective) compared to placebo taping, while placebo taping may only improve PPI in these patients. The short-term effect of KT over the low back region cannot improve lumbar proprioception when measured by active lumbar RE. Therefore, the findings do not support the hypothesis that lumbar taping would improve lumbar repositioning errors.

\begin{abstract}
Abbreviations
CLBP: Chronic Low Back Pain; SD: Standard Deviations; KT: Kinesio tape; CNS: Central Nervous System; RE: Repositioning Error;

EMG: Electromyographic; SF-MPQ: Short-Form McGill Pain Questionnaire; S/ A: Sensory and Affective; ODI: Oswestry Disability Questionnaire; PPI: Present Pain Intensity; VAS: Visual Analog Scale; ROM: Range of Motion; CE: Constant Error; AE: Absolute Error; NRS: Numerical Rating Scale; MDC: Minimal Detectable Change
\end{abstract}

\section{Acknowledgments}

The authors thank Dr. Hedayat Abbastabar for helping with data analysis and all participants.

\section{Authors' contributions}

SA and MRH were responsible for the study design. SA, NGH collected and prepared the data. OR and GHO analyzed the data. OR and SA were responsible for writing and redrafting the manuscript. All authors read and approved the final manuscript.

\section{Funding}

This study was supported by the School of Rehabilitation at Tehran University of Medical Sciences

\section{Availability of data and materials}

The datasets generated and analyzed during the current study are available from the corresponding author upon request.

\section{Ethics approval and consent to participate}

All participants signed a consent form, and the study was approved by the Human Ethics Committee at Tehran University of Medical Sciences, Tehran, Iran.

\section{Consent for publication}

Written informed consent was obtained from the participants for publication of their individual details and accompanying images in this manuscript. The consent form is held by the first author and is available for review by the Editor-in-Chief.

\section{Competing interests}

The authors declare that they have no competing interests.

\section{Author details}

'Department of Physiotherapy, School of Rehabilitation, Tehran University of Medical Sciences, (TUMS), Tehran, Iran. ${ }^{2}$ Postgraduate Department, School of Rehabilitation, Tehran University of Medical Sciences, International, Brain and Spinal Injury Research Center (BASIR), Institute of Neuroscirnce, P.O. Box: 111551683, Tehran, Iran. ${ }^{3}$ Rehabilitation Research Center, Department of Physical Therapy, School of Rehabilitation Sciences, Iran University of Medical Sciences, Tehran, Iran. ${ }^{4}$ Department of Public Health and Nursing, Faculty of Medicine and Health Sciences, Norwegian University of Science and Technology (NTNU), Trondheim, Norway.

Received: 17 March 2020 Accepted: 22 October 2020

Published online: 20 November 2020

\section{References}

1. Sarlegna FR, Sainburg RL. The roles of vision and proprioception in the planning of reaching movements. Progress in Motor Control: Springer; 2009. p. 317-35.

2. Proske U, Gandevia SC. The proprioceptive senses: their roles in signaling body shape, body position and movement, and muscle force. Physiol Rev. 2012;92(4):1651-97.

3. Burgess PR, Wei JY, Clark FJ, Simon J. Signaling of kinesthetic information by peripheral sensory receptors. Annu Rev Neurosci. 1982;5(1):171-87.

4. Tong MH, Mousavi SJ, Kiers H, Ferreira P, Refshauge $K$, van Dieen J. Is there a relationship between lumbar proprioception and low back pain? A systematic review with meta-analysis. Arch Phys Med Rehabil. 2017;98(1): 120-36. e2.

5. Solomonow M, Baratta R, Zhou BH, Shoji H, Bose W, Beck C, et al. The synergistic action of the anterior cruciate ligament and thigh muscles in maintaining joint stability. Am J Sports Med. 1987;15(3):207-13. 
6. O'Sullivan K, Verschueren S, Van Hoof W, Ertanir F, Martens L, Dankaerts W. Lumbar repositioning error in sitting: healthy controls versus people with sitting-related nonspecific chronic low back pain (flexion pattern). Man Ther. 2013;18(6):526-32.

7. Brumagne S, Cordo P, Lysens R, Verschueren S, Swinnen S. The role of paraspinal muscle spindles in lumbosacral position sense in individuals with and without low back pain. Spine (Phila Pa 1976). 2000;25(8):989-94

8. Descarreaux M, Blouin JS, Teasdale N. Repositioning accuracy and movement parameters in low back pain subjects and healthy control subjects. Eur Spine J. 2005;14(2):185-91.

9. Åsell M, Sjölander P, Kerschbaumer H, Djupsjöbacka M. Are lumbar repositioning errors larger among patients with chronic low back pain compared with asymptomatic subjects? Arch Phys Med Rehabil. 2006;87(9): 1170-6.

10. Newcomer K, Laskowski ER, Yu B, Larson DR, An KN. Repositioning error in low back pain. Comparing trunk repositioning error in subjects with chronic low back pain and control subjects. Spine (Phila Pa 1976). 2000;25(2):245-50.

11. Kurt EE, Buyukturan O, Erdem HR, Tuncay F, Sezgin H. Short-term effects of kinesio tape on joint position sense, isokinetic measurements, and clinical parameters in patellofemoral pain syndrome. J Phys Ther Sci. 2016;28(7): 2034-40.

12. Kase K, Jim W, Tsuyoshi K. Clinical therapeutic applications of the Kinesio taping method. 2nd ed. Tokyo; 2003. p. 19-39.

13. Halseth T, McChesney JW, DeBeliso M, Vaughn R, Lien J. The effects of Kinesio (TM) taping on proprioception at the ankle. J Sports Sci Med. 2004 3(1):1-7.

14. Murray $\mathrm{H}$. Effect of Kinesio taping on proprioception in the ankle. J Orthop Sports Phys Ther. 2001;31:A-37

15. Callaghan MJ, Selfe J, Bagley PJ, Oldham JA. The effects of patellar taping on knee joint proprioception. J Athl Train. 2002;37(1):19-24

16. Aytar A, Ozunlu N, Surenkok O, Baltacı G, Oztop P, Karatas M. Initial effects of kinesio ${ }^{\oplus}$ taping in patients with patellofemoral pain syndrome: a randomized, double-blind study. Isokinet Exerc Sci. 2011;19(2):135-42.

17. Bae SH, Lee JH, Oh KA, Kim KY. The effects of kinesio taping on potential in chronic low back pain patients anticipatory postural control and cerebral cortex. J Phys Ther Sci. 2013:25(11):1367-71.

18. Castro-Sanchez AM, Lara-Palomo IC, Mataran-Penarrocha GA, FernandezSanchez M, Sanchez-Labraca N, Arroyo-Morales M. Kinesio taping reduces disability and pain slightly in chronic nonspecific low back pain: a randomised trial. J Physiother. 2012;58(2):89-95.

19. Jassi FJ, Del Antonio T, Moraes R, George SZ, Chaves TC. Effects of functional taping compared with sham taping and minimal intervention on pain intensity and static postural control for patients with nonspecific chronic low back pain: a randomised clinical trial protocol. Physiotherapy. 2017;103(2):154-9.

20. Adelmanesh F, Arvantaj A, Rashki H, Ketabchi S, Montazeri A, Raissi G. Results from the translation and adaptation of the Iranian short-form McGill pain questionnaire (I-SF-MPQ): preliminary evidence of its reliability, construct validity and sensitivity in an Iranian pain population. Sports Med Arthrosc Rehabil Ther Technol. 2011;3(1):27.

21. Fairbank JC, Pynsent PB. The Oswestry disability index. Spine (Phila Pa 1976). 2000;25(22):2940-52 discussion 52.

22. Nelson NL. Kinesio taping for chronic low back pain: a systematic review. J Bodyw Mov Ther. 2016;20(3):672-81.

23. Keeley J, Mayer TG, Cox R, Gatchel RJ, Smith J, Mooney V. Quantification of lumbar function. Part 5: Reliability of range-of-motion measures in the sagittal plane and an in vivo torso rotation measurement technique. Spine (Phila Pa 1976). 1986;11(1):31-5.

24. Saur PM, Ensink FB, Frese K, Seeger D, Hildebrandt J. Lumbar range of motion: reliability and validity of the inclinometer technique in the clinical measurement of trunk flexibility. Spine (Phila Pa 1976). 1996;21(11):1332-8

25. Sadeghi R, Mosallanezhad Z, Nodehi-Moghadam A, Nourbakhsh MR, Biglarian A, Ezati K. The reliability of bubble inclinometer and tape measure in determining lumbar spine range of motion in healthy individuals and patients. Phys Treat Specific Phys TherJournal. 2015:5(3):137-44.

26. Lin $\mathrm{YH}$, Sun $\mathrm{MH}$. The effect of lifting and lowering an external load on repositioning error of trunk flexion-extension in subjects with and without low back pain. Clin Rehabil. 2006;20(7):603-8.

27. Faul F, Erdfelder E, Buchner A, Lang A-G. Statistical power analyses using $G^{*}$ power 3.1: tests for correlation and regression analyses. Behav Res Methods. 2009;41(4):1149-60.
28. Sullivan GM, Feinn R. Using effect size-or why the P value is not enough. J Grad Med Educ. 2012:4(3):279-82.

29. Polgar S, Thomas SA. Introduction to research in the health sciences E-book: Elsevier health sciences; 2011.

30. Added MAN, Costa LOP, de Freitas DG, Fukuda TY, Monteiro RL, Salomão EC, et al. Kinesio taping does not provide additional benefits in patients with chronic low back pain who receive exercise and manual therapy: a randomized controlled trial. J Orthop Sports Phys Ther. 2016;46(7):506-13.

31. Li Y, Yin Y, Jia G, Chen H, Yu L, Wu D. Effects of kinesiotape on pain and disability in individuals with chronic low back pain: a systematic review and meta-analysis of randomized controlled trials. Clin Rehabil. 2019;33(4):596606.

32. Al-Shareef AT, Omar MT, Ibrahim AH. Effect of Kinesio taping on pain and functional disability in chronic nonspecific low Back pain: a randomized clinical trial. Spine (Phila Pa 1976). 2016:41(14):E821-8.

33. Luz Júnior MA, Sousa MV, Neves LA, Cezar AA, Costa LO. Kinesio Taping ${ }^{\oplus}$ is not better than placebo in reducing pain and disability in patients with chronic non-specific low back pain: a randomized controlled trial. Braz J Phys Ther. 2015;19(6):482-90.

34. Uzunkulaoglu A, Gunes Aytekin M, Ay S, Ergin S. The effectiveness of Kinesio taping on pain and clinical features in chronic nonspecific low back pain: a randomized controlled clinical trial. Turk J Phys Med Rehabil. 2018;64(2): 126-32.

35. Macedo LB, Richards J, Borges DT, Melo SA, Brasileiro JS. Kinesio taping reduces pain and improves disability in low back pain patients: a randomised controlled trial. Physiotherapy. 2019;105(1):65-75.

36. Araujo AC. Do Carmo Silva Parreira P, Junior LCH, da Silva TM, da Luz Junior MA, da Cunha Menezes Costa $L$, et al. medium term effects of kinesio taping in patients with chronic nonspecific low back pain: a randomized controlled trial. Physiotherapy. 2018;104(1):149-51.

37. Parreira PCS, Costa LCM, Takahashi $R$, Junior LCH, da Luz Junior MA, da Silva TM, et al. Kinesio taping to generate skin convolutions is not better than sham taping for people with chronic non-specific low back pain: a randomised trial. J Physiother. 2014;60(2):90-6

38. Artioli DP, Bertolini GRF. Kinesio taping: application and results on pain: systematic review. Fisioterapia e Pesquisa. 2014;21(1):94-9.

39. Lumpkin EA, Caterina MJ. Mechanisms of sensory transduction in the skin. Nature. 2007:445(7130):858-65.

40. Abbasi S, Hadian MR, Olyaei GR, Ghotbi N, Bozorgmehr A, Rasouli O. Application of Various Methods of Lumbar Kinesio Taping on Pain and Disability in Patients with Chronic Low Back Pain: Narrative Review. Arch Neurosci. 2020;7(2):e99982.

41. Abbasi S, Rojhani-Shirazi Z, Shokri E. Garcia-Muro San Jose F. the effect of Kinesio taping on postural control in subjects with nonspecific chronic low back pain. J Bodyw Mov Ther. 2018;22(2):487-92.

42. Halseth T, McChesney JW, DeBeliso M, Vaughn R, Lien J. The effects of kinesio ${ }^{\mathrm{TM}}$ taping on proprioception at the ankle. J Sports Sci Med. 2004;3(1):1.

43. Paoloni M, Bernetti A, Fratocchi G, Mangone M, Parrinello L, Del Pilar CM, et al. Kinesio taping applied to lumbar muscles influences clinical and electromyographic characteristics in chronic low back pain patients. Eur J Phys Rehabil Med. 2011;47(2):237-44

44. Konishi Y. Tactile stimulation with kinesiology tape alleviates muscle weakness attributable to attenuation of la afferents. J Sci Med Sport. 2013; 16(1):45-8.

45. Felson DT, Gross KD, Nevitt MC, Yang M, Lane NE, Torner JC, et al. The effects of impaired joint position sense on the development and progression of pain and structural damage in knee osteoarthritis. Arthritis Rheum. 2009;61(8):1070-6.

\section{Publisher's Note}

Springer Nature remains neutral with regard to jurisdictional claims in published maps and institutional affiliations. 